\title{
Emergency Evacuation Simulation Model in a Large Building Based on GIS
}

\author{
Tian zhuo ${ }^{1}$, Libaicheng ${ }^{2}$ \\ Information teaching and Management Center \\ Jilin Agricultural University \\ Changchun, Jilin, China \\ xiaomarkshaoqing@163.com,lbc@163.com
}

Keywords:GIS; emergency evacuation; large building; visual simulation

\begin{abstract}
To meet the requirements of emergency evacuation in a large building, this paper introduces design and implementation of emergency evacuation simulation model in a large building based on GIS in detail. Firstly, three-tire architecture and functions of the simulation model are designed in the paper. Secondly, the key technologies of the model are emphasized. The base data of the model is the internal design map of a large hotel and the urban electronic map. The spatial analysis function of GIS is used to determine evacuation analysis of emergencies and disasters in a large building and achieve the optimal emergency evacuation plan for crowd. Research results showed the model improves the simulation precision and can provide effective service for emergency evacuation in a large building.
\end{abstract}

\section{Introduction}

With the rapid development of economy, public safety gets attention more and more. In the sites, such as shops, supermarkets, theaters, sports venues and other large buildings, the population density is very high. It will cause severe casualties if the evacuation is not in time when emergencies occur. In recent years, large buildings become more complex. How to evacuate the crowd rapidly and orderly has become an important responsibility of the government and the building management departments. To do large-scale practical exercises about evacuation of the crowd is very complicated and very difficult. So the simulation of the computer is the main research tool about evacuation method.

With the development of computer technology, the scientists have developed a variety of evacuation models using computer simulation technologies. However, at present, most of the computer simulations are numerical simulation ${ }^{[1]}$, which is the lack of realistic simulation. The evacuation simulation study of a large building involves a great deal of spatial geographic information. GIS (Geographic Information System) can achieve an effective management of spatial information and other types of information. Besides, GIS can achieve query, retrieval,statistical and computing of the geographic information data. Therefore, the emergency evacuation model of a large building based on GIS can achieve a realistic simulation to simulate the optimal emergency evacuation strategy at various emergency conditions, and provide scientific basis for relevant government departments in urban construction and in urban disaster prevention and reduction.

\section{Design of the Emergency Evacuation Simulation Model}

The emergency evacuation simulation model in a large building adopts three-tier architecture. The architecture is shown in Fig.1. Three-tier architecture is composed of access layer, function layer and data layer. 


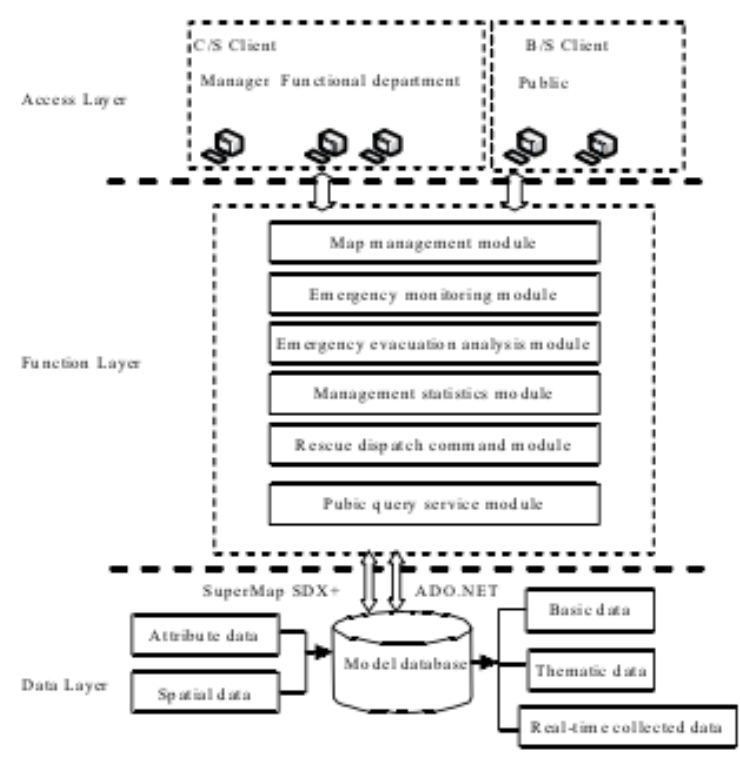

Figure 1. Architecture of model

Access Layer.Administrators of monitoring centers, other professionals of the relevant functional departments and the public have different access permissions to access the corresponding functional modules.

Function Layer.The function layer is the core of the model. The function layer is composed of map management module, emergency monitoring module, emergency evacuation analysis module, management statistics module ${ }^{[2]}$, rescue dispatch command module and public query services module. Here are its specific functions as follows.

1) Map management module: It includes map display,zoom, cruising, markup, ranging, area measurement, eagle-eye, layer control, projection selection etc.

2) Emergency monitoring module: It can be used to monitor the position, velocity, direction and the spread state of the emergencies in real-time and understand the disaster situation and timely feedback the emergency information and form the distribution map of the emergencies rapidly.

Data Layer.The data involved in the model includes basic data,thematic data, and real-time collected data. Basic data include administrative divisions in urban reas,transportation routes, water, settlements, topography, types of buildings, structures, as well as economic, demographic and related environmental data etc. Thematic data include the data inside the buildings and the lifeline engineering data etc. Real-time collected data include GPS data collected in real-time, the temperature and smoke information data collected in real-time by temperature sensors and smoke sensors. Oracle9i relational database is chosen as the physical database.

\section{The Key Technology of Model}

Analysis of disaster Zone. When users input the corresponding parameters, model can generate the different zones of disaster by the practical application of disaster spread model research including fire models, toxic gas models, explosion model, etc. When multiple disasters occur simultaneously, the model makes use of overlay analysis based on GIS in order that the crowd can be evacuated to safe zones. According to different influence of disaster, fire danger zones are divided into three levels: death zone, seriously injury zone and minor injury zone. Toxic gas disaster zones are divided into four levels:death zone, seriously injury zone, injury zone and inhalation reaction zone.

Evacuation Analysis.By the function of the crowd density map, decision makers can get a clear understanding of the distribution of crowd and can pay more attention to the zones ${ }^{[3]}$ where the densities are high because crowded sections may be resulted during evacuation process.

The time that person goes from the room to the staircase:When the density of crowd is low, the distance-controlled evacuation is used. GIS is used to establish the network topology and to make nodes at the intersections among the corridors. Person has different running speeds by reason of 
different densities.

$$
t_{m, 1}=\sum_{i=1}^{n} \frac{L_{m, i}}{v_{i}} \quad 1 \leq \mathrm{m} \leq \mathrm{k}
$$

In (1), $\mathrm{k}$ represents that there are $\mathrm{k}$ staircases in a large build, m represents staircase number, tm, 1 represents the time that person goes from the room to the entrance of the staircase $\mathrm{m}, \mathrm{Lm}, \mathrm{i}$ represents the corridor $\mathrm{i}$ on the shortest path to the entrance of the staircase $\mathrm{m}$ and vi represents the speed of the crowd flow in corridor i. Here the research results of S.M. Lo in Hong Kong are used[13], the relationship between the speed and the density is expressed with (2), where the density $\rho$ is the crowd number in unit area.

$$
v_{i(\rho)}=\left\{\begin{array}{lr}
1.4 & \rho \leq 0.75 \\
0.041 \rho^{2}-0.59 \rho+1.867 & 0.75<\rho \leq 4.2 \\
0 & \rho>4.2
\end{array}\right.
$$

\section{Simulation Test}

The operating environment of the model is as follows.Server configuration: CPU: Pentium (R) 3.0GHz, RAM: 2G;client Configuration: CPU: Pentium (R) 2.8GHz, RAM: 1G;browser: IE6.0; networking environment: LAN (100MEthernet). Fig. 2 and Fig. 3 are some simulation results. Fig2. is the simulation result after $15 \mathrm{~s}$ of fire. Fig. 3 is the simulation result after 40 s of fire. The black dots in the figures represent the evacuation personnel. Supposing all doors are opened and there are 5 staircase exits, according to the GIS buffer zone analysis and GIS overlap analysis by real-time information about temperature ${ }^{[4]}$ and smoke and spatial location information, serious injury zone will be extended to exit 4 of staircase after 19s of fire. So it means that exit 4 is in the high-risk zone. In order to prevent accidents, exit 4 should be closed after 19s of fire. Therefore,evacuation exits should be decreased from five to four after 19s of fire. The personnel ultimately evacuate ${ }^{[5]}$ to exit 1, exit 2,exit 3 and exit 5 in Fig. 3.

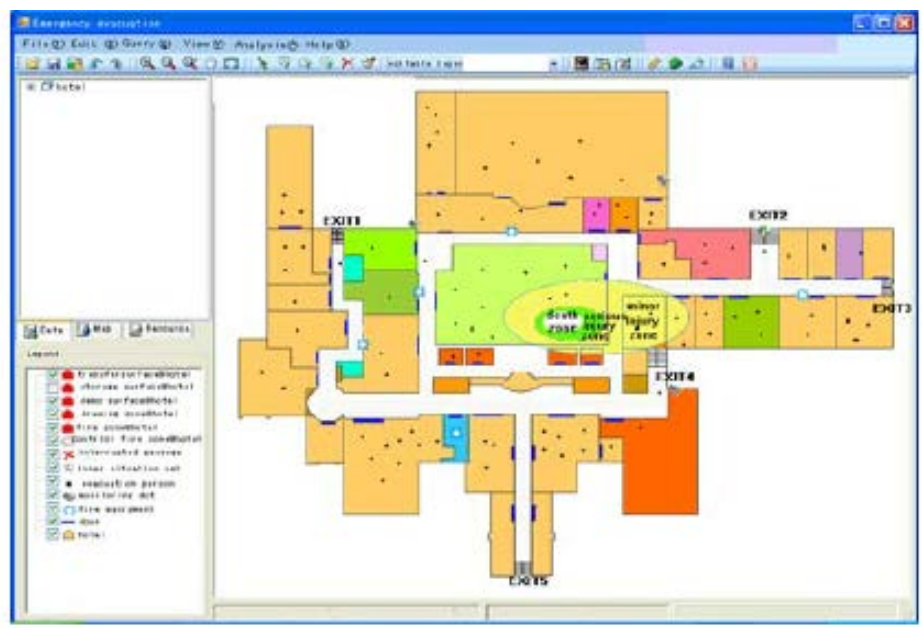

Figure 2. The evacuation simulation after 15 s of fire 


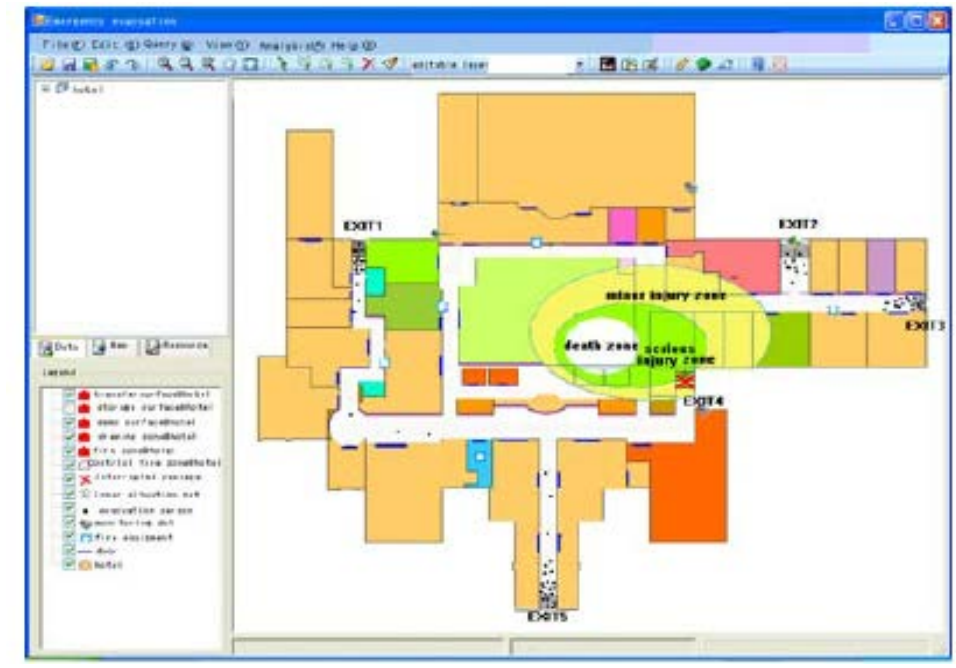

Figure 3. The evacuation simulation after 40s of fire

\section{Conclusion}

The base data of the model is internal design map of some hotel and the urban electronic map. The electronic surveillance, disaster spread simulation, GPS, GSM mobile communication, evacuation area analysis system and other technologies are used to achieve emergency evacuation of the personnel and establish the best evacuation plan.According to the related real-time data of wind speed, wind direction and evacuation speed, wind direction and evacuation speed, the powerful spatial analysis function of GIS is used to determine the affected zone of the dynamic spread of disasters to the mergency evacuation. Simulation examples show that the model improves simulation accuracy and has strong applicability and operability.

\section{References}

[1]H. Hu, X.K Yang, and X.M Liu,“Simulation on Emergency Evacuation Plan for 2008 Beijing Olympic Games,” in Proceedings of Engineering(ICTE), Chengdu, China, July22-24 2007, pp. 673-679.

[2]T.S. Shen, "ESM: A building evacuation simulation model," Building and Environment, vol. 40,no. 5, pp. 671-680, May 2005.S. Gwynne, E.R. Galea, P.J. Lawrence, and L. Filippidis, "Modelling occupant interaction with fire conditions using the building EXODUS evacuation model,” Fire Safety Journal, vol. 36, no. 4, pp. 327-357,April 2001.

[3]J. Shi, A. Ren, and C. Chen, “Agent-based evacuation model of large public building under fire conditions,”Automation in Construction,vol. 18, no. 3, pp. 338-347, May 2009.

[4]X.C Wu, A new generation of network-oriented GIS. Science Press,Beijing, 2001. R. Sieber, "Public participation geographic information systems: a literature review and framework," Annals of the Association of American Geographer, vol. 96, no. 3, pp. 491-507, March 2006.

[5] Z.G Wang, “An Occupant Evacuation Model for the Underground Shopping Mall during Fire Incidents, ” Fire Safety Science, vol.10, no.1, pp.57-62, January 2001. 